\title{
An innovative training based on robotics for older people with subacute stroke: study protocol for a randomized controlled trial
}

Elvira Maranesi ${ }^{1,2^{*}}$ (D), Roberta Bevilacqua ${ }^{2}$, Mirko Di Rosa ${ }^{3}$, Giuseppe Pelliccioni ${ }^{4}$, Valentina Di Donna ${ }^{5}$, Riccardo Luzi ${ }^{6}$, Micaela Morettini ${ }^{7}$, Agnese Sbrollini ${ }^{7}$, Elisa Casoni ${ }^{1}$, Nadia Rinaldi ${ }^{5}$, Renato Baldoni ${ }^{1}$,

Fabrizia Lattanzio ${ }^{2}$, Laura Burattini ${ }^{7}$ and Giovanni R. Riccardi ${ }^{1}$

\begin{abstract}
Background: Stroke is a leading cause of disability, injury, and death in elderly people and represents a major public health problem with substantial medical and economic consequences. The incidence of stroke rapidly increases with age, doubling for each decade after age 55 years. Gait impairment is one of the most important problems after stroke, and improving walking function is often a key component of any rehabilitation program. To achieve this goal, a robotic gait trainer seems to be promising. In fact, some studies underline the efficacy of robotic gait training based on end-effector technology, for different diseases, in particular in stroke patients. In this randomized controlled trial, we verify the efficacy of the robotic treatment in terms of improving the gait and reducing the risk of falling and its long-term effects.

Methods: In this single-blind randomized controlled trial, we will include 152 elderly subacute stroke patients divided in two groups to receive a traditional rehabilitation program or a robotic rehabilitation using G-EO system, an end-effector device for the gait rehabilitation, in addition to the traditional therapy. Twenty treatment sessions will be conducted, divided into 3 training sessions per week, for 7 weeks. The control group will perform traditional therapy sessions lasting $50 \mathrm{~min}$. The technological intervention group, using the G-EO system, will carry out $30 \mathrm{~min}$ of traditional therapy and 20 min of treatment with a robotic system. The primary outcome of the study is the evaluation of the falling risk. Secondary outcomes are the assessment of the gait improvements and the fear of falling. Further evaluations, such as length and asymmetry of the step, walking and functional status, and acceptance of the technology, will be carried.
\end{abstract}

Discussion: The final goal of the present study is to propose a new approach and an innovative therapeutic plan in the post-stroke rehabilitation, focused on the use of a robotic device, in order to obtain the beneficial effects of this treatment.

Trial registration: ClinicalTrials.gov NCT04087083. Registered on September 12, 2019

Keywords: Stroke patients, Gait training, End-effector gait rehabilitation

\footnotetext{
* Correspondence: e.maranesi@inrca.it

${ }^{1}$ Clinical Unit of Physical Rehabilitation, IRCCS INRCA, Ancona, Italy

${ }^{2}$ Scientific Direction, IRCCS INRCA, Ancona, Italy

Full list of author information is available at the end of the article
}

C C The Author(s). 2021 Open Access This article is licensed under a Creative Commons Attribution 4.0 International License, which permits use, sharing, adaptation, distribution and reproduction in any medium or format, as long as you give appropriate credit to the original author(s) and the source, provide a link to the Creative Commons licence, and indicate if changes were made. The images or other third party material in this article are included in the article's Creative Commons licence, unless indicated otherwise in a credit line to the material. If material is not included in the article's Creative Commons licence and your intended use is not permitted by statutory regulation or exceeds the permitted use, you will need to obtain permission directly from the copyright holder. To view a copy of this licence, visit http://creativecommons.org/licenses/by/4.0/ The Creative Commons Public Domain Dedication waiver (http://creativecommons.org/publicdomain/zero/1.0/) applies to the data made available in this article, unless otherwise stated in a credit line to the data. 


\section{Background}

According to data from "World Population Prospects: the 2017 Revision," the number of older persons is expected to more than double by 2050 and to more than triple by 2100, rising from 962 million globally in 2017 to 2.1 billion in 2050 and 3.1 billion in 2100 [1]. Globally, the number of persons aged 80 or over is projected to triple by 2050 [2].

Stroke is a leading cause of disability, injury, and death in elderly people and represents a major public health problem with substantial medical and economic consequences [3]. The incidence of stroke rapidly increases with age, doubling for each decade after aged 55. Among adults aged 35 to 44 years old, the incidence of stroke is 30 to 120 of 100,000 per year, and for those ages 65 to 74 , the incidence is 670 to 970 of 100,000 per year [4]. Annually, 15 million people worldwide suffer from stroke. Of these, 5 million die and another 5 million are left permanently disabled [5]. Three months after stroke, $20 \%$ of individuals remains wheelchair bound and $70 \%$ walks at reduced velocity [6]. Gait impairment is one of the most important problems after stroke and it is associated with difficulties in performing daily activities and in maintaining indoor and outdoor mobility. For this reason, improving walking function is often a key component of any rehabilitation program. To achieve this goal, a specific task-repetitive training seems the most promising one since, by increasing therapy dosage, intensity, and number of repetitions, the plasticity and the functional recovery are promoted [7-9]. To achieve this goal, gait machines were developed following exoskeleton $[10,11]$ or end-effector principles [12]. In the exoskeleton systems, there is a one-to-one correspondence between robots and human joints, and each single joint is guided along a preprogrammed trajectory. The end-effector systems use footplates or handles to generate a motion of the limb in space, as movements are generated from the most distal segment of the extremity and no alignment between patientrobot joints is required. On the basis of the support provided, the electromechanical devices can be also divided into inactive, passive, active-assisted, resistive, and interactive devices [13].

Several trials have been published regarding the use of these devices in stroke patients, but no differences were found between the two types of robotic gait machines [14]. Despite this evidence, some studies [15-18] underline the efficacy of robotic gait training based on endeffector technology, for different diseases. In particular, stroke patients, exposed to only robot-assisted endeffector-based gait training, showed significant improvements in global motor performance, gait endurance, balance and coordination, lower limb strength, and even spasticity.

Due to the incidence of the gait impairments after stroke and the low degree of acceptance of exoskeletons [19], we strongly believe that the efficacy of robotic rehabilitation treatments based on end-effector technology has to be deeply investigated.

\section{Study aims and objective}

This study aims to evaluate an innovative rehabilitation treatment based on robotics, for the older subacute stroke patients, designed to improve the gait and to reduce the risk of falling. The treatment involves the use of the G-EO system (Reha Technology, Switzerland), an end-effector technology that simulates floor walking and stair climbing (up and down). The study objectives are:

1. To evaluate the effect of the rehabilitation treatment on the decrease of falling risk, evaluating balance and gait performance, of the elderly subacute stroke patients, as a result of the use of the G-EO system, at the end of the treatment and at 6 months, 1 year, and 2 years of follow-up

2. To evaluate the effect of the gait improvement by the gait speed evaluation on rehabilitation treatment on the gait speed of the elderly subacute stroke patients, as a result of the use of the G-EO system, at the end of the treatment and at 6 months and 1 and 2 years of follow-up

3. To evaluate the effect of the rehabilitation treatment on the fear of falling of the elderly subacute stroke patients, as a result of the use of the G-EO system, at the end of the treatment and at 6 months and 1 and 2 years of follow-up

In addition, the study design will include using standardized questionnaires and instrumental gait analysis, in order to collect data on the improvements with a mix-method approach.

\section{Methods/design}

\section{Trial design}

This study is a single-blind (outcome assessors) randomized controlled trial. In particular, the single-blind research method consists in not letting the researcher know the type of rehabilitation path assigned to each participant. Each subject will be assigned an identification code that will be known only to the assessor who will carry out the recruitment, the end of treatment, and the follow-up assessments. A total of 152 elderly subacute stroke patients will be recruited and randomly divided into two groups, to receive a traditional rehabilitation program or a robotic rehabilitation using the G-EO system in addition to the traditional therapy. 
Assessments will be performed at baseline, at the end of treatment, and 6 months, 1 year, and 2 years from the end of the treatment.

\section{Study setting}

The study will be conducted at the Clinical Unit of Physical Rehabilitation, IRCCS INRCA, in the Ancona and Fermo branches, Italy. Assessments and treatments will be conducted in the robotics laboratories.

\section{Trial status}

The protocol version number is 1 in date July 15, 2019.

At the time of the submission of this study protocol, data collection is ongoing; the first patient was enrolled on July 13, 2020. We expect to finish recruiting in 2 years.

\section{Participants}

The inclusion criteria are:

- Aged 65 and over

- Capacity to consent

- Ischemic or hemorrhagic stroke within 3 months to the recruitment, proven by computerized axial tomography or nuclear magnetic resonance

- Functional Ambulation Category (FAC) score $\leq 2$

- Rankin Scale score $\leq 3$

- Complete communication and comprehension skills, assessed during the objective examination

- Ability to stand upright, supported or unsupported, for 1 min [20]

The exclusion criteria are:

- Failure to meet the inclusion criteria

- Concomitant participation in other studies

- Severe hypertonus of the hip, knee, and ankle of the paretic leg with a modified Ashworth scale score $\geq 3$

- Severe hip, knee, or ankle contracture or orthopedic problem affecting ambulation that would preclude passive range of motion of the paretic leg

- Deep vein thrombosis of the lower limbs

- Other cognitive, motor, and sensory deficits that negatively condition robotic training

- Treatment of spasticity of the lower limb within 3 months to the start of the study or during the study

- Lack of written informed consent

- Clinical Dementia Rating (CDR) score $\geq 3$

- Severe systemic diseases with life expectancy $<1$ year

- Patients unable to follow-up

\section{Sample size}

In the study of Tramontano et al. [21], the PerformanceOriented Mobility Assessment (POMA) was evaluated in two groups of stroke patients, the cases were treated through an intervention based on visual-spatial training, and the assessments were detected in 2 stages (baseline and follow-up).

POMA [22], a test widely used to assess walking ability and associated with equilibrium, was used to calculate the sample size [23]. Assuming a small effect size of $10 \%$ [24], a statistical power of $80 \%$, a significance level of 0.05 , a correlation among repeated measures of 0.5 , and that sphericity is exactly met, it is estimated that the overall sample size needed to capture this effect size is of 122 subjects, two groups, and 5 repeated assessments (a baseline and 4 follow-ups) in an ANOVA model within-between interactions. Even assuming a 20\% dropout rate, the total number required would be 152 subjects (76 for each arm). The GPower (Heinrich-HeineUniversität Düsseldorf, Düsseldorf, Germany) software was used to estimate this sample size.

For the secondary outcomes, we assumed an improvement of $12 \%$ and $15 \%$ for gait speed and fear of falling, respectively. These increases have been hypothesized on the basis of articles $[25,26]$ that identify an improvement in these parameters using a treatment comparable to that used in this protocol.

\section{Recruitment}

Patients will be selected by the outpatient department at the Clinical Unit of Physical Rehabilitation, IRCCS INRCA, in the Ancona and Fermo branches. These patients will be contacted to schedule a visit with the physician. Once the compliance with the inclusion and exclusion criteria of the study has been verified and the informed consent has been obtained in triplicate, the doctor will proceed with the baseline evaluation and with acquisition of gait assessment parameters through gait analysis at the Movement Analysis Laboratory of the Clinical Unit of Physical Rehabilitation of the Ancona branch.

A randomization technique based on a single sequence of random assignments will be used. A list of random numbers generated by the computer will be used and subjects will be assigned a number based on their order of inclusion in the study. The sequence will be saved to a password-protected spreadsheet. According to this technique, the 152 subjects will be randomly assigned to one of the 2 study groups: the control group and the technological intervention group. After the patient is assigned to one of the two groups, he will be given a code by which the physiotherapist will understand whether the patient should receive traditional or experimental treatment. The randomization was organized by 
using opaque envelopes: participating physicians are given randomly generated treatment allocations within sealed opaque envelopes. Once a patient has consented to enter a trial, an envelope is opened and the patient is then offered the allocated treatment group. The randomization schedule and coding of group allocations will not be accessible to the researcher conducting the assessments. According to the intention to treat (ITT) principle, subjects who were enrolled but withdrawn from the study will not be replaced. Researchers will be blinded to prevent observation bias. Statistical analyses will be conducted by a statistician, who will be blind to group allocation prior to analysis.

At the end of the treatment and after 6 months, 1 year, and 2 years, the patients will be contacted again to schedule subsequent follow-up visits and upgrades.

Recruitment will run from August 2019 to August 2021.

\section{Intervention}

For the study, post-hospitalization subjects will be taken into consideration, after 4 weeks from the hospitalization in the Clinical Unit of Physical Rehabilitation, IRCCS INRCA, in the Ancona and Fermo branches, who have already received the standard treatment. Twenty treatment sessions will be conducted, divided into 3 training sessions per week, for 7 weeks. The control group will perform traditional therapy sessions lasting $50 \mathrm{~min}$. The technological intervention group will carry out $30 \mathrm{~min}$ of traditional therapy and $20 \mathrm{~min}$ of treatment with a robotic system. Cardiac activity monitoring is planned during robotic treatments.

Individual participants must complete at least $80 \%$ of the sessions. Recovery of 3 sessions will be possible.

All patients included in the study will perform traditional rehabilitation treatments, consisted in:

- Appropriate positioning in bed and chair

- Neuromotor treatment for the trunk control recovery and movement in bed

- Exercises for verticalization, load balancing, and balance control from an upright position

- Walking training with correct use of aids

- Neuromotor treatment for the recovery of functional motility of the upper limb
- Improved autonomy in daily life activities with the use of aids, if necessary

The robotic treatment consists of using the G-EO system, an advanced robot-assisted, end-effector device in gait rehabilitation. The G-EO system allows to simulate both floor walking and stair climbing. The G-EO floor walking movement is generated by the motion of two footplates and allows to select different execution modality: active mode, active-assistive mode, and passive mode. The standard procedure consists of 5 sessions performed using the passive mode and 15 using the active-assistive mode. Taking into account that a personalized approach will be carried out, the therapy may be modified in based of patients' needs. Cardiac activity monitoring is planned during robotic treatments.

Adverse events, although unlikely, could be related to the use of technology such as falls and/or pain at knees or ankles. In this case, these effects are further minimized by the presence of the lashing.

\section{Outcomes}

All outcome measures follow a standardized operating procedure. Table 1 shows the primary outcome and the secondary outcomes with the expected result at the end of the treatment. The expected improvement was derived from the analysis of similar studies [24], collected for the evaluation of the sample size for each outcome.

Further evaluations will be carried out as follows:

- Length and asymmetry of the semi-step, through instrumental gait analysis

- Walking and functional status through the Functional Ambulation Category and Barthel Index scale

- Acceptance of the technology, through a UTAUT questionnaire

A summary of all data collected and when these are collected is provided in Table 2 .

\section{Mini-Mental State Examination (MMSE)}

The MMSE was designed as a clinical method for grading cognitive impairment. The score ranges from 0 to 30 : scores $\geq 24$ indicate normality, between 18 and 23 indicate mild cognitive impairment, between

Table 1 Outcomes and clinical assessments

\begin{tabular}{lll}
\hline Outcome(s) & Clinical assessment & Expected improvement at the end of treatment (\%) \\
\hline Primary: Decrease of falling risk & POMA & 10 \\
Secondary: Gait improvement (increase in walking speed) & Gait speed (gait analysis) & 12 \\
Secondary: Decrease of fear of falling & FES-I Short form & 15 \\
\hline
\end{tabular}

POMA Tinetti's Scale or Performance-Oriented Mobility Assessment, FES-/ Short Falls Efficacy Scale-International 
Table 2 Schedule of assessment and outcome measures

\begin{tabular}{|c|c|c|c|c|c|c|}
\hline Outcome & Clinical assessment & $\mathbf{R}$ & T1 & FW1 & FW2 & FW3 \\
\hline Cognitive state & Mini-Mental State Examination & $\checkmark$ & & & & \\
\hline Gait parameters & Functional Ambulation Category & $\checkmark$ & $\checkmark$ & $\checkmark$ & $\checkmark$ & $\checkmark$ \\
\hline Disability state & Rankin Scale & $\checkmark$ & & & & \\
\hline Cognitive state & CDR & $\checkmark$ & & & & \\
\hline Functional state & Barthel Index & $\checkmark$ & $\checkmark$ & $\checkmark$ & $\checkmark$ & $\checkmark$ \\
\hline Spasticity state & Modified Ashworth scale & $\checkmark$ & & & & \\
\hline Quality of life & SF-12 Health Survey & $\checkmark$ & $\checkmark$ & $\checkmark$ & $\checkmark$ & $\checkmark$ \\
\hline Sociodemographic characteristics & Check-list & $\checkmark$ & & & & \\
\hline Motor ability & Motricity index & $\checkmark$ & $\checkmark$ & $\checkmark$ & $\checkmark$ & $\checkmark$ \\
\hline Attitude to technology & Assistive Device Predisposition Assessment - Scala E & $\checkmark$ & & & & \\
\hline Fall risk & Scala di Tinetti & $\checkmark$ & $\checkmark$ & $\checkmark$ & $\checkmark$ & $\checkmark$ \\
\hline Gait parameters & Gait analysis + instrumental postural analysis & $\checkmark$ & $\checkmark$ & $\checkmark$ & $\checkmark$ & $\checkmark$ \\
\hline Fear of falling & Short Falls Efficacy Scale_-International & $\checkmark$ & $\checkmark$ & $\checkmark$ & $\checkmark$ & $\checkmark$ \\
\hline Acceptance of technology & Psychosocial Impact of Assisted Device Scale (PIADS) & & $\checkmark$ & & & \\
\hline
\end{tabular}

$R$ recruitment, $T 1$ end of treatment, FW1 first follow-up at 6 months since the end of treatment, FW2 second follow-up at 1 year since the end of treatment, FW3 third follow-up at 2 years since the end of treatment

11 and 17 average cognitive deficits, and scores $\leq 10$ severe cognitive impairments. The reported score is corrected for age and education [27].

\section{Rankin Scale (RA)}

It is a simple scale for the evaluation of the outcomes following the stroke. Reliability is well defined. The individual categories are essentially based on patient mobility. There are 6 grades of classification from 0 to 5, where 0 means independence [28].

\section{Barthel Index (BI)}

$\mathrm{BI}$ is an ordinal scale used to measure a subject's performance in everyday life activities. The index analyzes ten variables that describe the activities of daily life and mobility. Each item is assigned a score between 0 and 10 depending on the degree of patient's functionality: full, reduced, or no functionality. A high overall score is associated with a greater probability of being able to live at home independently after discharge from the hospital [29].

\section{Functional Ambulation Categories (FAC)}

The scale is used to classify the severity level of gait disturbances in neurological disorders. It provides a hierarchical classification from level 0 (impossible walking) to level 5 (no limitation) [30].

\section{Modified Ashworth scale (SA)}

The scale carries out the assessment of spasticity/hypertonus. The scores range from 0 to 4,0 no spasticity and 4 rigid limbs in flexion or extension [31].

\section{SF-12 Health Survey (SF-12)}

The SF-12 questionnaire was originally developed in the USA to provide a short alternative form to the SF-36 questionnaire. The SF-12 is composed of 12 items that produce two measurements related to two different aspects of health: physical health and mental health. The subject is asked to answer on how he feels and how he is able to carry out the usual activities, evaluating the current day and the 4 previous weeks [32].

\section{Tinetti's Scale or Performance-Oriented Mobility Assessment (POMA)}

Tinetti scale is a tool used to evaluate balance and gait performance. The test is used clinically to determine the mobility status of a subject or to assess changes in balance and gait time. The total POMA (POMA-T) consists of two sub-scales: the balance evaluation scale ("balance scale" or POMA-B) and the gait evaluation scale ("gait scale" or POMA-G). The maximum score is 28 points: in detail, the maximum score of the POMA-B is 16 , while for the POMA-G the maximum score is 12 [22].

\section{Motricity index (MI)}

The scale shows a significant correlation between lower limb scores and dynamometric measurements. The total score ranges from 0 to 100 . It provides 3 items with a score between 0 and 33: 0 no movement, 33 movement performed with normal force [33].

\section{Short Falls Efficacy Scale-International (FES-I-Short)}

The scale measures the "fear of falling." The scale can be self-administered or administered during the interview. The cutoffs for the fear of falling are divided as follows: 
a score between 7 and 8 indicates a low concern, between 9 and 12 a moderate concern, and between 14 and 28 a high concern [34].

\section{Psychosocial Impact of Assistive Devices Scale (PIADS)}

It is a self-completed questionnaire by the user and it assesses the impact that the device has on the person. Through 26 questions, it tries to detect how the device has brought about a perception of change with respect to one's availability for new experiences (6 questions), skills (ability to cope with daily activities and challenges-12 questions), and self-esteem (security and selfconfidence-8 questions). Every question is answered on a visual scale marked by -3 (the device has strongly limited my independence) to +3 (the device has greatly improved my independence) [35].

\section{Assistive Device Predisposition Assessment (ATDPA)}

The purpose of the tool is to assess user expectations about technological devices [36].

\section{Clinical Dementia Rating Scale (CDR)}

This questionnaire assesses the patient's dementia status. The CDR is a 5-point scale used to characterize six domains: memory, orientation, judgment and problem solving, business, home and hobby, and personal care [37].

\section{Gait analysis and instrumental postural analysis}

Gait analysis is the systematic study of human locomotion, augmented by instrumentation for measuring body movements, body mechanics, and the activity of the muscles [38]. Gait analysis is performed on the selected patients at the Gait Analysis Laboratory in the Department of Physical Rehabilitation at branch of IRCCS INRCA Ancona. Instrumented gait analysis is performed using BTS GAITLAB (BTS Bioengineering, Italy) system with six infrared cameras $(100 \mathrm{~Hz})$ and 2 force plates $(50 \mathrm{~Hz})$. The system is used to acquire both kinematic and kinetic data. Three-dimensional kinematic data are recorded with the help of 22 reflective infrared markers using Helen Hayes protocol [39]. The floor-mounted force plates are used to acquire the kinetic data. The subjects walked at a self-selected speed. The instrumental postural analysis studies the complex control system that must keep the center of gravity constantly in a balanced position. This analysis is carried out through an advanced system composed of a camera for video recording and a platform with 2 triaxial sensory plates (Podium, BTS Bioengineering, Italy). With this system, it is possible to simultaneously visualize the three components of force: vertical, antero-posterior, and lateral. It allows to evaluate, in augmented reality, the symmetry of the load and the trend of the pressure center.

\section{Data management}

Personal data collected during the trial will be handled and stored in accordance with the General Data Protection Regulation (GDPR) 2018. Use of the study data will be controlled by the principal investigator. All data and documentation related to the trial will be stored in accordance with applicable regulatory requirements and access to data will be restricted to authorized trial personnel.

\section{Data analysis}

The ITT approach will be adopted. Descriptive statistics will be used to describe the patients' characteristics at baseline, compare the POMA values, the FES-I scores, and the gait speed in the two groups. Continuous variables will be expressed as mean values \pm standard deviation. Categorical variables will be expressed as absolute values and relative frequencies. Independent samples' ttests and chi-squared test analyses will be used to compare both baseline characteristics and outcomes between groups. The primary efficacy analysis will be based on within-between group differences in the primary outcome measures (decrease in fall risk measured by POMA score) following 6 months of training with the G-EO system. The secondary outcome progression will be evaluated by comparing the measures of gait speed and FES-I scale from baseline to 6-month follow-up.

Comparisons for both primary and secondary outcomes will be repeated at each time point. Finally, repeated measures mixed models with interactions for each outcome will be performed in order to evaluate the effectiveness of the intervention. All tests for significance used a two-sided p-value of 0.05. Data were analyzed with STATA 15.1 (StataCorp, College Station, TX, USA).

\section{Discussion}

The aim of this protocol is to evaluate an innovative rehabilitation treatment of the elderly subacute stroke patients, designed to improve the gait and to reduce the risk of falling through the use of the G-EO system, an end-effector technology that simulates floor walking and stair climbing. Moreover, the study aims to evaluate the effect of the rehabilitation treatment on the balance and gait performance, on the gait speed, on the fear of falling, and on the quality of life, acceptance of technology, and improvement of functional status as results of the use of the G-EO system.

The target population of the study consists of stroke patients within 3 months from the event, who need physical assistance from another person to walk in the form of continuous or intermittent manual contact, or who cannot walk independently. A population with these characteristics was chosen because the scientific 
evidence shows that patients with highly impaired walking ability are able to have greater benefits from robotic rehabilitation than those patients who are already walking independently [40]. A total of 152 elderly subacute stroke patients will be recruited and randomly divided into two groups, to receive a traditional rehabilitation program or a robotic rehabilitation using the G-EO system in addition to the traditional therapy.

We will compare the results of the several outcomes (falling risk, gait improvement in terms of walking speed, length and asymmetry of the step, fear of falling, walking and functional status, and acceptance of the technology) between the two groups in order to verify the efficacy of the robotic treatment. The study considers three different follow-ups: at 6 months, 1 year, and 2 years from the end of the treatment to monitor the conservation of any improvements obtained.

In fact, the final goal of the present study is to propose a new approach and an innovative therapeutic plan in the post-stroke rehabilitation, focused on the use of robotic device, in order to obtain the beneficial effects of this treatment.

The dissemination program will involve peer-reviewed journal and national and international conferences. Moreover, the results will be disseminated to all participants.

\section{Abbreviations}

ATDPA: Assistive Technology Device Predisposition Assessment; Bl: Barthel Index; CDR: Clinical Dementia Rating Scale; FAC: Functional Ambulation Categories; FES-I-Short: Short Falls Efficacy Scale-International; GDPR: General Data Protection Regulation; ITT: Intention to treat; MI: Motricity index; MMSE: Mini-Mental State Examination; PIAD S: Psychosocial Impact of Assistive Devices Scale; POMA: Tinetti's Scale or Performance-Oriented Mobility Assessment; RA: Rankin Scale; SA: Modified Ashworth scale; SF-12: SF-12 Health Survey

\section{Acknowledgements}

The authors would like to thank Dr. Alberto Montesanto (University of Calabria, Cosenza - IT), who provided statistical assistance in the preparation of this article.

\section{Authors' contributions}

EM and RB led the design and writing of the pilot RCT protocol. GRR, VDD, and GP helped with the development of the participant identification plan and provided advice on other key study issues. EC, NR, and RB helped with the design of the intervention. MDR contributed to the calculation of the sample size and will lead the collection, management, and statistical analysis of the data. FL and RL contributed feedback on the trial design. MM, AS, and LB were responsible for manuscript draft revising. All the authors contributed important intellectual content to the written protocol and read and approved the final manuscript.

\section{Funding}

This research received no specific grant from any funding agency in the public, commercial, or not-for-profit sectors.

\section{Availability of data and materials}

The datasets generated, used, and analyzed during the trial and its preceding pilot trial are or will be available from the corresponding author upon reasonable request.

\section{Declarations}

\section{Ethics approval and consent to participate}

The study was approved by IRCCS INRCA Ethics Committee during the session of July 17, 2019, with identification code number 19014. Any protocol modifications will be notified to the same Ethics Committee and to other interested parties such as researchers and participants. For the latter, changes will be communicated by email. The principals of the Declaration of Helsinki and Good Clinical Practice guidelines will be adhered to. Participants in this study will provide written informed consent.

\section{Consent for publication}

Not applicable.

\section{Competing interests}

The authors declare that they have no competing interests and the study has not received external funding.

\section{Author details}

${ }^{1}$ Clinical Unit of Physical Rehabilitation, IRCCS INRCA, Ancona, Italy. ${ }^{2}$ Scientific Direction, IRCCS INRCA, Ancona, Italy. ${ }^{3}$ Unit of Geriatric

Pharmacoepidemiology, IRCCS INRCA, Ancona, Italy. ${ }^{4}$ Neurology Unit, IRCCS INRCA, Ancona, Italy. ${ }^{5}$ Clinical Unit of Physical Rehabilitation, IRCCS INRCA, Fermo, Italy. ${ }^{6}$ Medical Direction, IRCCS INRCA, Ancona, Italy. ${ }^{7}$ Cardiovascular Bioengineering Lab, Department of Information Engineering, Università Politecnica delle Marche, Ancona, Italy.

Received: 24 August 2020 Accepted: 4 June 2021

Published online: 14 June 2021

\section{References}

1. World Health Organization. The World Health Report 2002: reducing risks, promoting healthy life: World Health Organization; 2002.

2. United Nation, Department of Economic and Social Affairs. World population prospects: the 2017 revision; 2017.

3. Truelsen T, Piechowski-Jozwiak B, Bonita R, Mathers C, Bogousslavsky J, Boysen G. Stroke incidence and prevalence in Europe: a review of available data. Eur J Neurol. 2006;13(6):581-98. https://doi.org/10.1111/j.1468-1331.2 006.01138.x

4. Ovbiagele B, Nguyen-Huynh MN. Stroke epidemiology: advancing our understanding of disease mechanism and therapy. Neurotherapeutics. 2011; 8(3):319-29. https://doi.org/10.1007/s13311-011-0053-1.

5. Truelsen T, Mahonen M, Tolenen H, Asplund K, Bonita R, Vanuzzo D, et al. Trends in stroke and coronary heart diseases in the WHO MONICA Project. Stroke. 2003;34(6):1346-52. https://doi.org/10.1161/01.STR.0000069724.361 73.4D.

6. Taveggia G, Bordoni A, Mulé C, Villafane $\mathrm{JH}$, Negrini S. Conflicting results of robot-assisted versus usual gait training during postacute rehabilitation of stroke patients: a randomized clinical trial. Int J Rehabil Res. 2016;39(1):2935. https://doi.org/10.1097/MRR.0000000000000137.

7. Molteni F, Gasperini G, Cannaviello G, Guanziroli E. Exoskeleton and endeffector for upper and lower limbs rehabilitation: narrative review. Arch Phys Med Rehabil. 2018;10:\$174-88.

8. Apte $\mathrm{S}$, Plooij M, Vallery $\mathrm{H}$. Influence of body weight unloading on human gait characteristics: a systematic review. J Neuroeng Rehabil. 2018;15(1):53. https://doi.org/10.1186/s12984-018-0380-0.

9. Van Kammen K, Boonstra A, Reinders-Messelink H, den Otter R. The combined effects of body weight support and gait speed on gait related muscle activity: a comparison between walking in the Lokomat exoskeleton and regular treadmill walking. Plos ONE. 2014;9:9.

10. Veneman JF, Kruidhof R, Hekman EE, Ekkelenkamp R, Van Asseldonk EH, van der Kooij H. Design and evaluation of the LOPES exoskeleton robot for interactive gait rehabilitation. IEEE Trans Neural Syst Rehabil Eng. 2007;15(3): 379-86. https://doi.org/10.1109/TNSRE.2007.903919.

11. Banala SK, Kim SH, Agrawal SK, Scholz JP. Robot assisted gait training with active leg exoskeleton (ALKEX). IEEE Trans Neural Syst Rehabil Eng. 2009; 17(1):2-8. https://doi.org/10.1109/TNSRE.2008.2008280.

12. Hesse $\mathrm{S}$, Waldner A, Tomelleri C. Innovative gait robot for the repetitive practice of floor walking and stair climbing up and down in stroke patients. J Neuroeng Rehabil. 2010;7(1):30. https://doi.org/10.1186/1743-0003-7-30. 
13. Chen G, Chan CK, Guo Z, Yu H. A review of lower extremityassistive robotic exoskeletons in rehabilitation therapy. Crit Rev Biomed Eng. 2013;41(4-5): 343-63. https://doi.org/10.1615/CritRevBiomedEng.2014010453.

14. Schwartz I, Meiner Z. Robot-assisted gait training in neurological patient: who may benefit? Ann Biomed Eng. 2015;43(5):1260-9. https://doi.org/10.1 007/s10439-015-1283-x.

15. Mazzoleni S, Focacci A, Franceschini M, Waldner A, Spagnuolo C, Battini E, et al. Robot-assisted end-effector-based gait training in chronic stroke patients: a multicentric uncontrolled observational retrospective clinical study. NeuroRehabil. 2017;40(4):483-92. https://doi.org/10.3233/NRE-161435.

16. Galli M, Cimolin V, De Pandis MF, Le Pera D, Sova I, Albertini G, et al. Robotassisted gait training versus treadmill training in patients with Parkinson's disease: a kinematic evaluation with gait profile score. Funct Neurol. 2016; 31(3):163-70. https://doi.org/10.11138/fneur/2016.31.3.163.

17. Sale P, Stocchi F, Galafate D, De Pandis MF, Le Pera D, Sova I, et al. Effects of robot assisted gait training in progressive supranuclear palsy (PSP): a preliminary report. Front Hum Neurosci. 2014:8:207.

18. Esquenazi A, Lee S, Wikoff A, Packel A, Toczylowski T, Feeley J. A comparison of locomotor therapy interventions: partial-body weightsupported treadmill, Lokomat, and G-EO training in people with traumatic brain injury. Am J Phys Med Rehabil. 2017;9:839-46.

19. Babaiasl M, Mahdioun SH, Jaryani P, Yazdani M. A review of technological and clinical aspects of robot-aided rehabilitation of upper-extremity after stroke. Disabil Rehabil Assist Technol. 2016;11(4):263-80. https://doi.org/1 0.3109/17483107.2014.1002539.

20. Ng MFW, Tong RKY, Li LSW. A pilot study of randomized clinical controlled trial of gait training in subacute stroke patients with partial body-weight support electromechanical gait trainer and functional electrical stimulation six-month follow-up. 2008. https://doi.org/10.1161/STROKEAHA.107.495705.

21. Tramontano M, Dell'Uomo D, Cinnera AM, Luciani C, Di Lorenzo C, Marcotulli M, et al. Visual-spatial training in patients with sub-acute stroke without neglect: a randomized, single-blind controlled trial. Funct Neurol. 2019;34(1):7-13

22. Tinetti ME. Performance-oriented assessment of mobility problems in elderly patients. J Am Geriatr Soc. 1986;34(2):119-26. https://doi.org/10.1111/j.15325415.1986.tb05480.x.

23. Faber MJ, Bosscher RJ, van Wieringen PC. Clinometric properties of the performance-oriented mobility assessment. Phys Ther. 2006;86(7):944-54. https://doi.org/10.1093/ptj/86.7.944.

24. Meurisse GM, Bastien GJ, Schepens B. The step-to-step transition mode: a potential indicator of first-fall risk in elderly adults? PLoS One. 2019;14(8): e0220791. https://doi.org/10.1371/journal.pone.0220791.

25. Shulman LM, Katzel LI, Ivey FM, Sorkin JD, Favors K, Anderson KE, et al. Randomized clinical trial of 3 types of physical exercise for patients with Parkinson disease. JAMA Neurol. 2013;70(2):183-90. https://doi.org/10.1001/ja maneurol.2013.646.

26. Cakit BD, Saracoglu M, Genc H, Erdem HR, Inan L. The effects of incremental speed-dependent treadmill training on postural instability and fear of falling in Parkinson's disease. Clin Rehabil. 2007;21(8):698-705. https://doi.org/10.11 77/0269215507077269.

27. Folstein MF, Folstein SE, McHugh PR. Mini-mental state. A pratical method for grading the cognitive state of patients for the clinician. J Psychiatric Res. 1975;12(3):189-98. https://doi.org/10.1016/0022-3956(75)90026-6.

28. Van Swieten JC, Koudstaal PJ, Visser MC, Schouten HJA, van Gijn J. Interobserver agreement for the assessment of handicap in stroke patients. Stroke. 1988;19(5):604-7. https://doi.org/10.1161/01.STR.19.5.604.

29. Mahoney Fl, Barthel DW. Functional evaluation: the Barthel Index. Md State Med J. 1965;14:61-5.

30. Holden MK, Gill KM, Magliozzi MR. Gait assessment for neurologically impaired. Standards for outcome assessment. Phys. Ther. 1986;66(10):15309. https://doi.org/10.1093/ptj/66.10.1530

31. Bohannon RW, Smith MB. Interrater reliability of a modified Ashworth scale of muscle spasticity. Phys Ther. 1987;67(2):206-7. https://doi.org/10.1093/ptj/ 67.2.206.

32. Ware JE, Kosinski M, Keller SD. SF-12: how to score the SF-12 physical and mental health summary scales. 3rd ed. Lincoln, Rl: QualityMetric Incorporated; 1998.

33. Demeurisse G, Demol O, Robaye E. Motor evaluation in bascular hemiplegia. Eur. Neurol. 1980;19(6):382-9. https://doi.org/10.1159/000115178.

34. Ruggiero C, Mariani T, Gugliotta R, Gasperini B, Patacchini F, Nguyen HN, et al. Validation of the Italian version of the falls efficacy scale international
(FES-I) and the SHORT FES-I in community dwelling older persons. Arch Gerontol Geriatrics. 2009;39(S):211-9.

35. Jutai J, Day H. Psychosocial Impact of Assistive devices Scale (PIADS). Technol Disabil. 2002;14(3):107-11. https://doi.org/10.3233/TAD-2002-14305.

36. Scherer MJ, Cushman LA. Measuring subjective quality of life following spinal cord injury: a validation study of the assistive technology device predisposition assessment. Disabil Rehabil. 2001;23(9):387-93. https://doi. org/10.1080/09638280010006665

37. Morris JC. Clinical Dementia Rating: a reliable and valid diagnostic and staging measure for dementia of the Alzheimer type. Int Psychogeriatrics. 1997:9(S1):173-6. https://doi.org/10.1017/S1041610297004870.

38. Levine DF, Richards J, Whittle M. Whittle's gait analysis: Elsevier Health Sciences; 2012. ISBN 978-0702042652

39. Davis RB, Ounpuu S, Tyburski D, Gage JR. A gait analysis data collection and reduction technique. Hum Movement Sci. 1991;10(5):575-87. https://doi. org/10.1016/0167-9457(91)90046-Z.

40. Lo K, Stephenson M, Lockwood C. Effectiveness of robotic assisted rehabilitation for mobility and functional ability in adult stroke patients: a systematic review. JBI Database System Rev Implement Rep. 2017;15(12): 3049-91. https://doi.org/10.11124/JBISRIR-2017-003456.

\section{Publisher's Note}

Springer Nature remains neutral with regard to jurisdictional claims in published maps and institutional affiliations.
Ready to submit your research? Choose BMC and benefit from:

- fast, convenient online submission

- thorough peer review by experienced researchers in your field

- rapid publication on acceptance

- support for research data, including large and complex data types

- gold Open Access which fosters wider collaboration and increased citations

- maximum visibility for your research: over $100 \mathrm{M}$ website views per year

At $\mathrm{BMC}$, research is always in progress.

Learn more biomedcentral.com/submissions 